\title{
Near-optimal Integration of Magnitude in the Human Parietal Cortex
}

\author{
Hannah Tickle ${ }^{1}$, Maarten Speekenbrink ${ }^{1}$, Konstantinos Tsetsos ${ }^{2}$, \\ Elizabeth Michael ${ }^{2}$, and Christopher Summerfield ${ }^{2}$
}

\begin{abstract}
Humans are often observed to make optimal sensorimotor decisions but to be poor judges of situations involving explicit estimation of magnitudes or numerical quantities. For example, when drawing conclusions from data, humans tend to neglect the size of the sample from which it was collected. Here, we asked whether this sample size neglect is a general property of human decisions and investigated its neural implementation. Participants viewed eight discrete visual arrays (samples) depicting variable numbers of blue and pink balls. They then judged whether the samples were being drawn from an urn in which blue or pink predominated. A participant who neglects the sample size will integrate the ratio of balls on each array, giving equal weight to each sample. However, we found that
\end{abstract}

\section{INTRODUCTION}

Decisions often involve integration of evidence from multiple sources. Optimal choices are made when information is weighted by the trustworthiness (or reliability) of each source. When human sensorimotor behavior is refined through experience, it often resembles that of an ideal observer (Pouget, Beck, Ma, \& Latham, 2013; Kording, 2007). For example, humans pointing toward a target assign more weight to prior knowledge about its location when sensory evidence is indistinct, as an ideal observer should (Kording \& Wolpert, 2004). Humans and monkeys can learn to weight a train of symbolic cues in direct proportion to the informativeness with which they predict a rewarded response (Gould, Nobre, Wyart, \& Rushworth, 2012; Wyart, de Gardelle, Scholl, \& Summerfield, 2012; Yang \& Shadlen, 2007). When visual and haptic cues offer potentially conflicting information about the size of an object, visual information is less influential when corrupted by noise (Ernst \& Banks, 2002). These findings have prompted the claim that humans and monkeys have evolved to make optimal decisions, that is, those that account for the relative uncertainty associated with each source of choice-relevant information.

However, human choices are not always optimal. For example, when asked to evaluate hypothetical scenarios involving numerical magnitudes, humans often make

${ }^{1}$ University College London, ${ }^{2}$ University of Oxford human behavior resembled that of an optimal observer, giving more credence to larger sample sizes. Recording scalp EEG signals while participants performed the task allowed us to assess the decision information that was computed during integration. We found that neural signals over the posterior cortex after each sample correlated first with the sample size and then with the difference in the number of balls in either category. Moreover, lateralized beta-band activity over motor cortex was predicted by the cumulative difference in number of balls in each category. Together, these findings suggest that humans achieve statistically near-optimal decisions by adding up the difference in evidence on each sample, and imply that sample size neglect may not be a general feature of human decision-making.

biased or inaccurate responses (Griffin \& Tversky, 1992). Consider the problem of estimating whether men outnumber women on a university degree course. The approximate male-female ratio from a small seminar group is a less reliable estimator than that from a large lecture class, because our confidence in an estimate should be determined by its standard error, which is inversely related to $n$, that is, to the sample size. Given both observations (small seminar vs. large lecture), an optimal solution to this problem - which is given by combining the binomial probability associated with each sample-will afford the larger sample more weight; simply averaging the two ratios may lead to a biased decision. However, when confronted with problems of this nature, humans are excessively reliant on the relative balance of evidence, overlooking the information about sample size and drawing erroneous conclusions from data (Griffin \& Tversky, 1992; Kahneman, Slovic, \& Tversky, 1982). Nor is this phenomenon limited to binomial estimation problems; when asked to judge whether the average height of a group of humans exceeds a fixed value, humans disregard whether the group is composed of 10, 100, or 1000 individuals. Even researchers who use statistics regularly to evaluate data have been observed to display this "sample size neglect" (Tversky \& Kahneman, 1971).

Why, then, do humans account for the reliability of information in some situations and not in others? One possibility is that simple sensorimotor decisions (e.g., pointing 
toward a target) and higher-level cognitive judgments (e.g., estimating gender ratios) rely on qualitatively distinct computations. For example, optimal performance might be possible when uncertainty arises early in processing (e.g., from sensory noise), but neglect of sample size might occur when judgments are limited by more cognitive factors. Alternatively, sample size neglect might not be a ubiquitous phenomenon but might depend instead on contextual factors, such as the format in which the decision information is provided. For example, sample size neglect might occur when decision problems are presented as descriptions of hypothetical scenarios, but not when participants learn to make decisions in an experience-dependent fashion, that is, through feedback that reveals whether a classification judgment was correct or incorrect (Hertwig \& Erev, 2009).

Here, we asked whether humans performing a psychophysical task display sample size neglect or whether they integrate information about numerical magnitudes optimally. Our task was an expanded judgment task, variants of which have previously been used to interrogate information integration during perceptual decision-making (Wyart, de Gardelle, et al., 2012; Yang \& Shadlen, 2007). Our approach thus investigates sample size neglect via an experimental framework that has been widely used to understand the neural and computational mechanisms by which perceptual inputs are integrated and categorized (Gold \& Shadlen, 2007). Observers viewed a succession of eight discrete visual events ("samples") in which a variable number of pink and blue balls were displayed and subsequently decided whether they had been drawn from a larger pool of predominantly pink or predominantly blue balls. Our initial question was whether humans gave more credence to samples that contained more balls. For example, consider two different samples each offering a 2:1 ratio of blue-pink balls, one with three balls (two blue, one pink) and one with 12 balls (eight blue, four pink). If humans exhibit a sample size bias, their choices would reflect the integration of these ratios of evidence (i.e., they would weight samples with 3 and 12 balls equally). However, if humans are optimal, they will give more weight to samples with more balls.

We initially predicted that humans would show sample size neglect during integration of magnitude information. However, we found strong support for the opposing hypothesis: Human choices were "near optimal," in that their choices resembled those that might be made by an ideal observer (in this case, one who was calculating the binomial probability of the dots on each sample being drawn from one urn as opposed to the other). In the light of this finding, we turned our attention to understanding how this behavior might be achieved at the neural and computational levels. In our task, two simple strategies would allow participants to arrive at the nearoptimal solution. First, participants could add up the absolute evidence for either response, by integrating the blue and pink dots independently and comparing the resulting tallies (we call this the tally model; it is related to the "race" model of perceptual decision-making; Smith \& Vickers, 1988; Vickers, 1979). Alternatively, participants could add up the relative evidence for either choice, by integrating the difference in the number of blue or pink dots on each sample (we call this the difference model; it is related to the drift diffusion model of perceptual choice; Ratcliff \& McKoon, 2008). Because the behavioral data did not allow us to arbitrate among these possibilities, we turned to neural recordings and measured the scalp EEG while participants performed the task. Although we observed a correlate of the absolute number of pink or blue dots over posterior electrode sites, this was rapidly followed by a correlate of their relative difference. Over centroparietal sites previously implicated in perceptual categorization, we observed stronger correlates of both the momentary and integrated difference signal. Together, these findings suggest that humans solved the task by accumulating the relative difference in magnitudes of evidence on each sample, allowing them to perform the task near-optimally via a computationally tractable strategy.

\section{METHODS \\ Participants}

Fifty-four participants (31 women, 23 men) were recruited. All reported normal or corrected-to-normal vision and no history of neurological or psychiatric disorders and gave written informed consent in accordance with local ethical guidelines. Participants for the behavioral pilot $(n=15)$ and control experiment $(n=19)$ received $£ 10$ in compensation, and those undergoing EEG ( $n=$ 20) received $\$ 25$. Data from four EEG participants were excluded (before preprocessing) because of excessive movement and/or electrical interference, leaving $n=$ 16 for that experiment. For behavioral analyses, we included all pilot and valid EEG participants (total $n=31$ ).

\section{Task Design and Stimuli}

In both behavioral and EEG experiments, participants completed a probabilistic decision-making task (Figure 1). On each trial, one of two virtual "urns" was pseudorandomly selected: either one with a 60:40 predominance of pink balls (50\% trials) or one with a 60:40 predominance of blue balls ( $50 \%$ trials). Eight draws (with replacement) of $2,4,6,8,10$, or 12 balls were made from the relevant urn. The blue and pink balls drawn were represented as dots in a circular aperture on each of the eight sample screens for each trial. After viewing the eight samples, participants indicated whether the samples were drawn from the predominantly pink urn or predominantly blue urn.

The visual stimuli were presented using the Psychophysics 3 Toolbox (Brainard, 1997) running in MATLAB (The MathWorks, Natick, MA) on a 17-in. CRT monitor with 
Figure 1. Experimental protocol. Each trial commenced with a blank black screen for $1 \mathrm{sec}$, followed by a central fixation cross for $1 \mathrm{sec}$. Eight draws (with replacement) of 2 , $4,6,8,10$, or 12 balls were made from a virtual "urn" either with a $60: 40$ predominance of pink balls ( $50 \%$ trials) or with a 60:40 predominance of blue balls ( $50 \%$ trials), and each draw was represented on screen as colored dots within a circular aperture. Each screen also contained between one and six white distractor dots and was displayed for $150 \mathrm{msec}$ with 150-msec ISI. These target screens were preceded and followed by a forward and backward mask created in an identical fashion, except that the colored dots were orange and green, and participants were instructed to ignore them. At the end of the sample series, participants saw a screen prompting them to respond with a keyboard press. Feedback was given on each trial, with a high-pitched $(800 \mathrm{~Hz})$ tone for correct response and low-pitched $(400 \mathrm{~Hz})$ for incorrect response. resolution of $1024 \times 768$ pixels and a refresh rate of $60 \mathrm{~Hz}$ All stimuli were presented on a black background. Participants viewed the stimuli in a quiet darkened room approximately $70 \mathrm{~cm}$ from the screen. Before the experiment began, instructions were presented to participants on screen, including a visual cue indicating the ratio of pinkblue balls in the two urns. Each trial began with a white central fixation cross with lines of length 60 pixels (for $1000 \mathrm{msec}$ ), followed by a blank screen (1000 msec). Subsequently, participants viewed a forward mask, eight sequentially occurring sample screens, and a backward mask, each occurring for 150 msec with 150-msec ISI. Each sample screen consisted of an array of blue, pink, and white dots, each 20 pixels in diameter, randomly spatially distributed (minimum separation of 10 pixels) among 71 possible locations within a circular aperture of 300-pixel diameter. The number of blue and pink dots was determined by randomly drawing between 2 and 12 balls from one of two virtual urns as described above. White balls were distracters, which served to decorrelate decision information from low-level visual input; one to six white balls were randomly added to each sample. The mask screens were identical to sample screens except that the colored balls were green and orange, and participants were instructed to ignore them. These screens helped ensure that participants were not unduly swayed by the first or last sample (i.e., avoided perceptual primacy/recency effects).

After the presentation of the samples, participants indicated via a key press whether they thought the balls on that trial were drawn from the mainly pink urn ("m" key, with the right hand) or from the mainly blue urn (" $\mathrm{z}$ " key, with the left hand). Auditory feedback was given with a high tone $(800 \mathrm{~Hz})$ for correct answers and a low tone $(400 \mathrm{~Hz})$ for incorrect answers. Before they began the task, participants were given clear instructions, a visual representation of the decision problem and urns, and two practice trials with feedback.

The behavioral pilot differed from the EEG experiment in three ways: (i) the presentation time and ISI were $250 \mathrm{msec}$ each rather than $150 \mathrm{msec}$, (ii) each participant completed 288 rather than 570 trials, and (iii) the forward and backward masks were omitted.

\section{Statistically Optimal Solution}

Let us denote the number blue and pink balls on sample $k$ as $d 1_{k}$ and $d 2_{k}$, respectively, with $n_{k}=d 1_{k}+d 2_{k}$. The probability of drawing a blue ball from Urn 1 (predominantly blue) was $p$, and the probability of drawing a blue ball from Urn 2 (predominantly pink) was $1-p$. The converse was true for pink balls. By design, $p=.6$.

The statistically optimal solution to the task is given by the integration of binomial probabilities. For any sample $k$, the likelihood that the balls are drawn from the blue urn is given by

$$
p_{k}^{\prime}=B\left(d 1_{k}, n_{k}, p\right)
$$

The optimal decision rule is defined by whether the sum of the log likelihood ratios of each sample coming from either urn is greater or less than zero. On any sample $k$, the evidence or optimal decision update (DU) for or against each response can be quantified as the loglikelihood ratio:

$$
\log \left(\frac{B\left(d 1_{k}, n_{k}, p\right)}{B\left(d 1_{k}, n_{k}, 1-p\right)}\right)
$$




\section{Models of Evidence Integration}

We considered three models of evidence integration and choice that human observers could be using to solve the task: a suboptimal model and two models that arrived at the statistically optimal solution via two qualitatively different computations. Thus, these latter two make identical behavioral predictions but different predictions about the neural activity that would accompany each sample. We first defined the suboptimal model, one in which evidence was not weighted by sample size. In the ratio

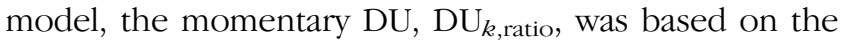
log ratio of blue-to-pink dots:

$$
\mathrm{DU}_{k, \text { ratio }}=\log \left(\frac{d 1_{k}}{d 2_{k}}\right)
$$

Thus, the ratio model ignores the sample size: For example, the same value of DU is obtained for $d 1_{k}=1$ and $d 2_{k}=3$ (where the number of balls $n_{k}=4$ ) as for $d 1_{k}=$ 3 and $d 2_{k}=9$ (where $n_{k}=12$ ). Choices were made according to whether the decision variable (DV), $\mathrm{DV}_{\text {ratio, }}$ was greater or less than zero:

$$
\mathrm{DV}_{\text {ratio }}=\sum_{k=1}^{8} \mathrm{DU}_{k, \text { ratio }}
$$

This policy accounts for the behavior of participants in the experiments of Tversky and colleagues described above.

Next, we considered two models that are formally equivalent to the statistically optimal solution but that solve the task via more biologically plausible mechanisms. We call these the difference and tally models. The difference model computes the difference between the number of blue and pink dots on each sample and adds up these differences to form the DV. Choices are then made according to whether this DV is greater or less than zero:

$$
\begin{gathered}
\text { DU } U_{\text {difference }}=d 1_{k}-d 2_{k} \\
\mathrm{DV}_{\text {difference }}=\sum_{k=1}^{8} \mathrm{DU}_{k, \text { difference }}
\end{gathered}
$$

This model predicts that brain signals accompanying each sample should correlate with the difference between the number of pink dots and the number of blue dots, that is, $d 1_{\mathrm{k}}-d 2_{\mathrm{k}}$.

By contrast, the tally model adds up the number of blue and pink dots in each stream without computing their difference. This model thus computes two momentary DVs for each sample, and the choice is made according to whether the sum of blue dots (DU1) exceeds the sum of pink dots (DU2) or vice versa (i.e., according to the sign of the DV):

$$
\begin{aligned}
& \mathrm{DU} 1_{\text {tally }}=d 1_{k} \\
& \mathrm{DU} 2_{\text {tally }}=d 2_{k}
\end{aligned}
$$

$$
\mathrm{DV}_{\text {tally }}=\sum_{k=1}^{8} \mathrm{DU} 1_{\text {tally }}-\sum_{k=1}^{8} \mathrm{DU} 2_{\mathrm{tally}}
$$

This model predicts that brain signals accompanying each sample should correlate with the total number of blue dots and the total number of pink dots, that is, $d 1_{k}$ and $d 2_{k}$.

Thus, although the quantity DV on which decisions are based is identical for the difference and tally models, because they arrive at this DV via different computations they make different predictions about the neural activity that will accompany each sample. The difference and tally models are related (but not identical) to the diffusion and race models often used to model RTs in psychophysical tasks (Ratcliff \& McKoon, 2008; Smith \& Vickers, 1989; Vickers, 1979).

For comparison with human data, $\mathrm{DV}_{\text {model }}$ was corrupted with values drawn from a Gaussian distribution, with mean of zero and a standard deviation of $\sigma$, before being used to generate categorical (model) choices. The noise parameter $\sigma$ was fit to group performance separately for the tally and difference models versus the ratio model but yielded very similar values (in log units: 0.51 for the ratio model and 0.59 for the tally and difference models). Note that varying this parameter simply changed overall model performance without affecting the qualitative pattern of results. The distributions of DV associated with the tally and difference models were very similar; each was roughly normally distributed and ranged from -3 to 3 in $\log$ units.

\section{Behavioral Analyses}

We compared human and model performance in a number of ways. First, we plotted psychometric functions to envisage how the probability of responding pink, $p$ (pink), varied as a function of the DVs predicted by the ratio, difference, and tally models (each binned into deciles). Second, we used probit regression model to estimate the influence that each of the sample positions (from first to last in the sequence) had on choice and plotted how $p$ (pink) was predicted by the number of blue and pink balls on each of the eight samples, as follows:

$$
p(\text { pink })=\phi\left\lceil b+\sum_{k=1}^{8} w_{k} \cdot d 1_{k}+w_{k} \cdot d 2_{k}\right\rceil
$$

where $\phi$ denotes the standard normal cumulative distribution function.

Finally, we tested how the influence of each sample on choice differed as a function of the total number of dots, $d 1_{k}+d 2_{k}=n_{k}$. The ratio model predicts that all samples should have the same weight, irrespective of $n_{k}$. The difference and tally models predict that the weight carried by each sample should increase as a function of $n_{k}$, in line with the statistically optimal (binomial) process. On each trial, we sorted the eight samples according to $n_{k}$, from lowest to highest, denoting the rank of each sample $j$, and 
used probit regression to calculate the coefficients that best mapped $\mathrm{DU}_{\text {ratio }}$ onto the choices made by humans and the various computational models:

$$
p(\text { pink })=\phi\left[b+\sum_{j=1}^{8} w_{j} \cdot \mathrm{DU}_{\mathrm{ratio}, j}\right]
$$

The logic of this analysis is that if humans are integrating the ratio of evidence, then the resulting coefficients should be flat across different values of $n_{k}$ (i.e., over the ranks $j$ ), whereas if they are performing (near-) optimally, then the coefficients should grow with $j$.

\section{EEG Acquisition and Preprocessing}

A Neuroscan (El Paso, TX) EEG system with NuAmps digital amplifiers was used to record EEG signals from $32 \mathrm{Ag}-\mathrm{AgCl}$ electrodes, located at FP1, FPz, FP2, F7, F3, Fz, F4, F8, FT7, FC3, FCz, FC4, FT8, T7, C3, Cz, C4, T8, TP7, CP3, CPz, CP4, TP8, P7, P3, Pz, P4, P8, POz, O1, Oz, and $\mathrm{O} 2$, plus four additional electrodes used in a bipolar montage as horizontal and vertical EOGs and two electrodes located at the mastoids used as reference. The electrode impedances were kept below $10 \mathrm{k} \Omega$. EEG signals were recorded at a sampling rate of $1 \mathrm{kHz}$ and highpass filtered online at $0.1 \mathrm{~Hz}$.

Preprocessing was carried out using the EEGLAB toolbox for MATLAB (Delorme \& Makeig, 2004) and custom scripts. The data were downsampled to $250 \mathrm{~Hz}$ and epoched from $1 \mathrm{sec}$ before the onset of the first sample to $6 \mathrm{sec}$ after it, thereby covering the entire trial of eight samples (including masks). The data were then visually inspected to remove trials containing nonstereotypical artifacts and to identify electrodes showing electrical artifacts and therefore requiring interpolation. After this, the data were bandpass filtered between 1 and $30 \mathrm{~Hz}$ and rereferenced to the average signal over all electrodes.

An independent component analysis was then conducted using EEGLAB, and the resulting independent component analysis components were visually inspected for artifacts, particularly stereotypical artifacts such as blinks and sustained high-frequency noise. Trials with artifacts were excluded from all further analysis, leaving an average of 492 (range $=378-544$ ) trials per participant, each consisting of eight overlapping stimulus events (sample onsets).

Unless otherwise stated, we report statistical tests on EEG data averaged across occipital (O1, O2, and Oz) and parietal (P3, Pz, P4, and POz) electrode sites. We chose this approach because previous studies have identified dissociable patterns of activity over occipital and parietal electrodes in discrete-sample integration tasks (Wyart, Nobre, \& Summerfield, 2012). To correct for multiple comparisons across time, we used a nonparametric cluster correction technique, implementing a familywise error (FWE) with an alpha of $\alpha=.05$ (Maris \& Oostenveld, 2007).

\section{EEG Analyses: Encoding}

We used EEG to investigate how the quantities predicted by the difference and tally models were encoded in neural signals, with a view to arbitrating between them, using a "model-based" approach to the analysis of brain imaging data. Both of these models made identical predictions about choice behavior but made different predictions about the neural activity that would accompany each sample. Rather than calculating ERPs, we estimated how decision information was encoded in EEG signals using a single-trial approach. This "encoding" methodology involves using parametric predictors (such as $\mathrm{DU}_{\text {model }}$ ) within a general linear regression model to predict the sample-to-sample variability in the EEG signal, at successive time points ( -100 to $700 \mathrm{msec}$ ) surrounding the onset of each sample.

First, we took model-predicted quantities $\left|\mathrm{DU}_{\text {difference }}\right|$ and $\left|\mathrm{DU}_{\text {ratio }}\right|$ and standardized these by $z$ transformation. Using rectified DUs $\left|\mathrm{DU}_{k}\right|$ ensures that we identify neural signals that encode absolute decision information, not those that favor one choice over another (i.e., blue vs. pink), as we were aiming to elucidate the nature of the mechanism rather than the nature of the choice per se. (Consider, as an analogy, random dot kinematogram motion discrimination tasks: The neural signal of interest from EEG recordings is one that correlates with the coherence level of the dots, i.e., decision information independent of direction of motion, rather than one which correlates with the extent to which the information favors leftward vs. rightward motion). We then regressed these quantities in a point-by-point fashion against the singletrial EEG activity after each corresponding sample. The resulting parameter estimates (slopes of the best-fitting regression line) provide an estimate of how strongly the EEG signal (at each time point over the course of the sample) varies with these model-predicted quantities. This thus allowed us to assess the difference in neural processing of reliability-weighted and non-reliabilityweighted information. Although the analysis epochs after each sample are overlapping, we took careful steps to ensure that the correlation between the variables of interest between adjacent samples was minimized. Thus, because the decision information provided by each sample is sufficiently uncorrelated, responses to adjacent stimulus events (samples) can be disambiguated, much as they can in parametric event-related functional neuroimaging designs (Josephs, Turner, \& Friston, 1997).

Subsequently, we repeated this procedure, including in the same regression the two quantities that are predicted by the difference and tally models, respectively: the total number of blue balls and the total number of pink balls (tally model) and the absolute difference in blue/pink balls (difference model). The aim of this analysis was to determine which of the two models was best able to account for the neural activity; including these predictors in the same regression ensured that they competed 
for unshared variance, allowing us to determine whether neural signals scaled more faithfully with the tally of evidence or the difference of evidence.

In all of these analyses, decision information from the preceding and succeeding samples was included as additional nuisance covariates. This helped ensure that the resulting parameter estimates reflected neural encoding on the current sample and were not corrupted by decision information from adjacent samples that overlapped in time with the epoch. This step, combined with the fact that the partial correlation between adjacent samples was very low as described, meant that we could be confident that the encoding analysis avoided confounding the influence of future and preceding samples. The resulting parameter estimates (slopes) for each time point $(-100 \mathrm{msec}$ prestimulus to +700 msec post-stimulus) were then averaged across samples and entered into a second-level statistical analysis for comparison at the group level. Regions of time (and space, i.e., electrodes) where these curves deviate reliably from zero across the cohort indicate where decision information is reliably encoded in neural signals. This process is also detailed in our earlier publications using this method (Wyart, Nobre, et al., 2012).

\section{EEG Analyses: Decoding}

In a subsequent analysis step, we assessed how the strength of the relationship between decision information and neural signals assessed above (EEG encoding) predicted participants' choices. This analysis step is closely related to the calculation of "choice probabilities" of single-cell recording data (Nienborg \& Cumming, 2010) and to an analysis of the psychophysiological interaction between behavioral and neural variables in fMRI analysis (Gitelman, Penny, Ashburner, \& Friston, 2003). Here and in previous publications, we have called this analysis "EEG decoding" (Wyart, Myers, \& Summerfield, 2015; Wyart, Nobre, et al., 2012) because it allows us to quantify how fluctuations in EEG encoding of DU are "decoded" in downstream brain structures and consequently manifest in choice. Such an analysis involves the use of multivariate parametric regression to quantify the extent of the modulatory influence of the EEG signal on the relationship between $\mathrm{DU}_{\text {model }}$ and choice. A decoding analysis allows us to see whether samples that are encoded with higher-than-average strength (in other words, with positive residual variance) are more predictive of choice than those which are encoded with lower-than-average strength; if they are, we would see a clear decoding curve (i.e., a significant effect of the residual EEG signal on choice). This method of estimating how the single-trial relationship between input (psychological variable) and brain activity (physiological variable) predicts choices allows a more direct measurement of how brain activity mediates the link between stimulus inputs and the weight or influence that a sample of information wields over choices.
To calculate these "decoding" curves, we took the time course of the unexplained variance (residuals) of the regression of $\mathrm{DU}_{\text {tally }}$ and $\mathrm{DU}_{\text {difference }}$ on the EEG signal. We then entered this quantity, $r$, into a probit regression, alongside decision information, as a predictor of participants' choices for each sample $k$ and time point $t$ :

$$
\begin{aligned}
p(\text { pink })= & \phi\left[b+\sum_{k=1}^{8} w_{k} \cdot \mathrm{DU}_{k, \text { model }}\right. \\
& \left.\left.+\sum_{k=1}^{8} w_{k, t} \cdot \mathrm{DU}_{k, \text { model }} \times r_{k, t}\right)\right]
\end{aligned}
$$

Once again, averaging across samples and participants afforded a grand average and the opportunity to conduct group-level statistics. Positive deviations of $r$ from zero indicate times at which brain activity not only scaled with decision information but did so more strongly than on average (i.e., the gain of encoding of a particular sample was above average in comparison with the overall encoding curve). If this stronger neural encoding of the DU results in a stronger effect of the DU on choice, then this should show as a significant positive interaction between $r$ and DU, as reflected in the weight $w_{k, t}$. The methods used here have been used successfully in previous articles from our laboratory (Wyart et al., 2015; Wyart, de Gardelle, et al., 2012).

\section{EEG: Lateralized Beta-Band Activity}

On the basis of previous studies, which have shown that oscillatory activity in the beta-band range accompanies the buildup of information to a decision threshold, we investigated the encoding of momentary and cumulative decision information in time-frequency transformed signals. We measured how signed DUs (DU $\mathrm{D}_{\text {tally }}$ and $\mathrm{DU}_{\text {difference }}$ ) and the corresponding signed DVs were encoded in lateralized beta-band activity $(\sim 10-40 \mathrm{~Hz})$, using a comparable technique to the encoding regressions above, in which these quantities were regressed together against relative lateralized single-trial spectral power over the motor cortex in 10 logarithmically spaced frequency bands between 9 and $43 \mathrm{~Hz}$. The response made with the left index finger always corresponded to "blue," whereas the right index response always corresponded to "pink." Thus, the use of signed DUs for analyzing lateralized beta-band activity is crucial in this analysis because, unlike the signals interrogated in previous encoding analyses, here, the two alternatives (pink vs. blue) map onto putative neural signals that can be disambiguated at the whole-brain level using scalp EEG (i.e., hemispherically lateralized patterns of beta-band desynchronization over the motor and premotor cortices). For each participant, we computed the interhemispheric difference in beta activity at lateral central electrodes by subtracting the spectral log power of $(\mathrm{CP}+\mathrm{CP} 3)$ from $(\mathrm{C} 4+\mathrm{CP} 4)$. 


\section{Control Analyses}

In a further effort to determine that our results from the main experiments were not being driven by low-level visual properties of the stimuli, we took the number of pink, blue, and white balls, respectively, on each sample and standardized these by $z$ transformation. We then asked whether behavioral choice was significantly influenced by the white dots and whether neural signals encoded more strongly those quantities that were decision relevant (pink and blue balls) relative to those that were irrelevant (white balls).

Previous studies that have focused on the neural representation of number have found a parietal ERP that reflects the difference in magnitude (i.e., the total number of stimuli) between one group of dots and the next (Piazza \& Izard, 2009; Piazza, Izard, Pinel, Le Bihan, \& Dehaene, 2004). To control for similar effects in our data, we regressed the absolute difference in total number of dots (blue + pink + white) between one sample and the next against the EEG signal (using the regression methods described above, including samples 2-8 in the stream). In particular, we were interested in whether any decisionrelated signals observed in our experiment could be trivially explained by previously described neural adaptation to number.

\section{Behavioral Control Experiment}

In the experiments described above, all dots were equally sized, and so the number of dots was correlated with the pixel area of the colored dots on the display screen. Thus, one question that arises is whether choices are driven principally by low-level visual properties of the stimuli, that is, the area (number of pixels) that the colored dots take up on screen, or by the number of dots per se. To arbitrate among these possibilities, we conducted a further behavioral experiment in which we varied the size of the pink and blue dots from sample to sample. This ensured that the number of dots and the number of pixels favoring each choice were decorrelated.

The design (including timing of stimuli and number of trials) of this behavioral control task, which was conducted on a new cohort of 19 participants, was identical to the first EEG experiment except for the size and position of the dot array. Dots were randomly spatially distributed (minimum separation of 10 pixels) among 46 possible locations within a circular aperture of 500-pixel diameter. The diameter of the blue dots on each sample was randomly selected from a range of $11-45$ pixels (35 possible sizes), and the same process was applied independently to both the pink dots and the white dots on each sample, thus ensuring that the pixel area of each color separately on each sample, as well as the total filled pixel area, was decorrelated from the respective number of dots (Pearson's correlation $\sim .65$ ).
This control allowed us to regress both the number of dots and the size of the dots on each sample against choice, thereby allowing us to determine whether sample size exerted an influence over and above the physical size of the dots on screen.

\section{RESULTS}

\section{Behavior}

Participants chose the correct urn on $83 \pm 6 \%$ of trials, with RTs averaging $407 \mathrm{msec}$. After the addition of performance-limiting noise to the DV, all three models achieved comparable accuracy to humans ( $85 \%$ for the tally and difference models and $83 \%$ for the ratio model) and were able to predict psychometric functions well. We calculated how participants' choices varied as a function of the $z$-transformed DVs predicted by the tally and difference models and the ratio model, $D V_{\text {difference, }}$ $\mathrm{DV}_{\text {tally }}$, and $\mathrm{DV}_{\text {ratio. }}$. The models performed equivalently (Figure 2A). The tally and difference models behaved in line with the optimal solution, and both (as described) made equivalent predictions with the exception of noise.

We next used probit regression on the behavioral data to estimate the impact that the number of pink and blue balls in each sample (1-8) had on choice, as a function of its serial position. As expected, "pink" choices were predicted positively by the number of pink balls and negatively by the number of blue balls (Figure 2B); this analysis suggested that all the samples contributed at least in part to the decision (all gray bars deviated significantly from 0 ).

Finally, to test our main hypothesis, we determined whether participants accounted for sample size (number of dots) when making decisions. To this end, we used probit regression to estimate the impact that each sample had on choice, ranked not by its position but by the total number of colored dots, $n_{k}\left(=d 1_{k}+d 2_{k}\right)$, that is, by its overall reliability. We used $\mathrm{DU}_{\text {ratio }}$, the $\mathrm{DU}$ as calculated by the ratio model, as a predictor. This ensured that the resulting coefficients for observers who did not weight information by sample size would be flat over ranks of $n_{k}$, whereas an observer who weighted information by reliability would show a profile of steadily increasing weights (note that a mathematically equivalent alternative would be to use the statistically optimal solution as a predictor, in which case an ideal observer would show a flat profile of weights, whereas those for an observer who integrated the ratio of evidence would decline with $n$ ). Consequently, for the ratio model (Figure 2C, orange points), the weights did not vary with ranks of $n_{k}$, but the weight given to each sample ranked by $n_{k}$ grew steadily for both the models that arrived at the statistically optimal solution (tally model, purple points; difference model, green points). Again, the values predicted by these latter two models are equivalent to the statistically optimal solution, with any residual variability because of the noise term $\sigma$. 
Figure 2. Behavioral results and model predictions. (A) Probability of selecting "pink" as predicted by the DVs for the ratio model (orange dots) and the two reliability weighted models (tally model, purple crosses; difference model, green crosses); a DV below zero corresponds to responding "blue" and above zero corresponds to a "pink" response. (B) Impact (beta coefficient) of each sample on "pink" choices, ranked by serial position (1-8), as a function of the number of pink balls (first eight bars) and the number of blue balls (last eight bars) in the sample. Estimates were generated using probit regression. Gray bars show human data and orange-/ purple-/green-colored crosses show predictions of the ratio/ tally/difference models, respectively. (C) Weights (beta coefficients) given to each sample as ranked by sample size (smallest to largest) in evidence integration, calculated using probit regression. Gray bars correspond to human behavior; colored crosses show model estimates for weight given to the ranked samples. The ratio model, given that it does not reliably weight the samples, is flat across ranks. In all figures, bars show SEM.

Critically, the impact that each sample wielded over choices for humans depended on the sample size, as it did for the statistically optimal solution and the two models that approximated it; this was confirmed by an ANOVA on the weights over ranks, $F(7,240)=51.6$, $p<.001$. The beta weight in the human data for the largest samples was significantly higher than that of the smallest samples: $t(30)=13.1, p<.001$. In the subsequent neural analyses, we seek to distinguish which of the two approximations of the optimal (binomial) solution best describes human brain activity during performance of the task. The reasoning behind this approach is that it is implausible that neurons explicitly compute binomial probabilities but rather derive the solution via a computationally tractable mechanism, such as those described by both the tally and difference models. Furthermore, it allows us to tease apart the difference and tally models that make indistinguishable behavioral predictions.

\section{EEG: Encoding of DU in Broadband Occipital and Parietal Signals}

How did humans achieve near-optimal performance on the task? Although the tally and difference models both derive the statistically optimal solution with predictions that match human behavior, they make different predictions about the quantities that are computed en route to a decision. We thus analyzed the link between decision information, neural signals, and choices to provide an insight into the mechanisms by which humans were making decisions.

First, we sought to correlate EEG activity with the DU (i.e., the momentary information conveyed by each sample) predicted by each of the models. Initially, we focused on comparing the ratio model with the difference model, only the latter of which predicts reliability-weighted behavior. To this end, we regressed $\left|\mathrm{DU}_{\text {difference }}\right|$ and $\left|\mathrm{DU}_{\text {ratio }}\right|$ separately against the singletrial EEG data for each sample and averaged over the resulting coefficients at each post-stimulus time point. The use of rectified predictors allows us to identify neural signals that correlate with the absolute decision information, rather than favoring one choice over another (e.g., pink over blue). Standardization ensured that the resulting coefficients were directly comparable. Consistent with participants' behavioral tendency to act as if they were weighting the evidence by its reliability (Figure 2A), the response to $\left|D U_{\text {difference }}\right|$ was significantly greater than that to $\left|D_{\text {ratio }}\right|$ (Figure 3A and B). In other words, behavioral data indicate that humans pursue a near-optimal strategy, and neural data suggest that they do so by integrating reliability-weighted evidence rather than only the ratio of evidence on each sample.

Subsequently, we asked whether humans performed near-optimally by (i) adding up the differences in numbers of balls and deciding whether this quantity was greater or less than zero (difference model) or (ii) adding 
up the total information in each stream in parallel and deciding which was greater (tally model). The former account predicts that participants compute $\mathrm{DU}_{\text {difference }}$ on each sample; the latter account predicts that only $\mathrm{DU}_{\text {tally }}$ is updated on a sample-by-sample basis. We thus entered $\left|\mathrm{DU} 1_{\text {tally }}\right|,\left|\mathrm{DU} 2_{\text {tally }}\right|$, and $\left|\mathrm{DU} \mathrm{U}_{\text {difference }}\right|$ as competitive predictors of the EEG signal at each scalp electrode and time point from $100 \mathrm{msec}$ preceding sample onset to $700 \mathrm{msec}$ after its onset (see Methods). We observed that the neural variance accounted for by $\left|D U_{\text {difference }}\right|$ in parietal electrodes outweighed that accounted for by $\left|\mathrm{DU} 1_{\text {tally }}\right|$ and $\left|\mathrm{DU} 2_{\text {tally }}\right|$, in that the beta weights were larger for the former and reached significance for longer, with a first negative deflection peaking at $230 \mathrm{msec}$ and a second positive deflection at $470 \mathrm{msec}$ after stimulus onset (Figure 3D). It is important to note that these curves are not ERPs but single-trial estimates of the encoding of decision information by brain activity. Interestingly, the same regression in occipital electrodes (Figure 3C) showed an initial negative deflection for $\left|\mathrm{DU} 1_{\text {tally }}\right|$ and $\left|\mathrm{DU} 2_{\text {tally }}\right|$ followed $150 \mathrm{msec}$ later by a negative deflection for $\left|D U_{\text {difference }}\right|$ (Figure 3C). One might expect, even under the difference model, that there would be an early representation of the total number of pink and blue dots, as this quantity is required to calculate the difference. Thus, our interpretation of this finding is that the initial encoding of the absolute information is followed by the emergence of the decision-relevant quantity DU $U_{\text {difference. }}$

Repeating the above analysis at each electrode provided a topography of encoding of $\left|\mathrm{DU}_{\text {difference }}\right|$ and $\left|\mathrm{DU}_{\text {tally }}\right|$ across the scalp, which we collapsed into bins of $100 \mathrm{msec}$ (Figure 4; note that the two quantities DU $1_{\text {tally }}$ and DU $2_{\text {tally }}$ are combined for ease of presentation given their similarity). The spatial distribution of the resulting weights can be interpreted as the spatial topography of the underlying decision-related component in the EEG signal. The resulting patterns of significant activation were in accordance with the results from the regressions above, with the response to $\left|\mathrm{DU}_{\text {difference }}\right|$ (bottom row) outweighing that to $\left|\mathrm{DU}_{\text {tally }}\right|$ (top row). In other words, these neural observations suggest that participants use a strategy that involves encoding the difference in information provided by each sample. This allows them to derive the near-optimal solution, weighting information by its reliability.

\section{EEG: Decoding of DU in Broadband Parietal Signals}

The encoding regressions suggested that more of the neural variance was accounted for by the difference model, which encoded the relative evidence on each sample, than by the tally model, which encoded the absolute evidence.
Figure 3. Neural encoding curves. (A and B) Curves showing correlation between the predictions of the ratio model (orange curve) and the difference model (green curve), which approximates the binomial (optimal) solution, with EEG data after each sample. Shaded areas in the curves denote SEM. The bars at the top show periods from stimulus onset in which the curves consistently deviate significantly from zero across participants for difference (green) and ratio (orange) models. (C and D) Correlation between EEG signals and the DUs predicted by the tally model (blue and pink ball totals, blue and pink curves) and the difference model (blue balls-pink balls, green curve) Shaded areas in the curves denote SEM. The bars at the top show periods from stimulus onset in which the curves consistently deviate significantly from zero across participants for difference (green bar) and tally (blue and pink bars) models. Note that, for occipital electrodes, there is a period of significant activation corresponding to the tally model after stimulus onset, followed by significant activation corresponding to the difference model around $200 \mathrm{msec}$, as though all evidence is being processed initially followed by the formation of the decision-relevant difference signal. For all panels, statistics were computed using a nonparametric cluster-correction technique implementing an FWE correction with an alpha of $\alpha=.05$ (see Methods).

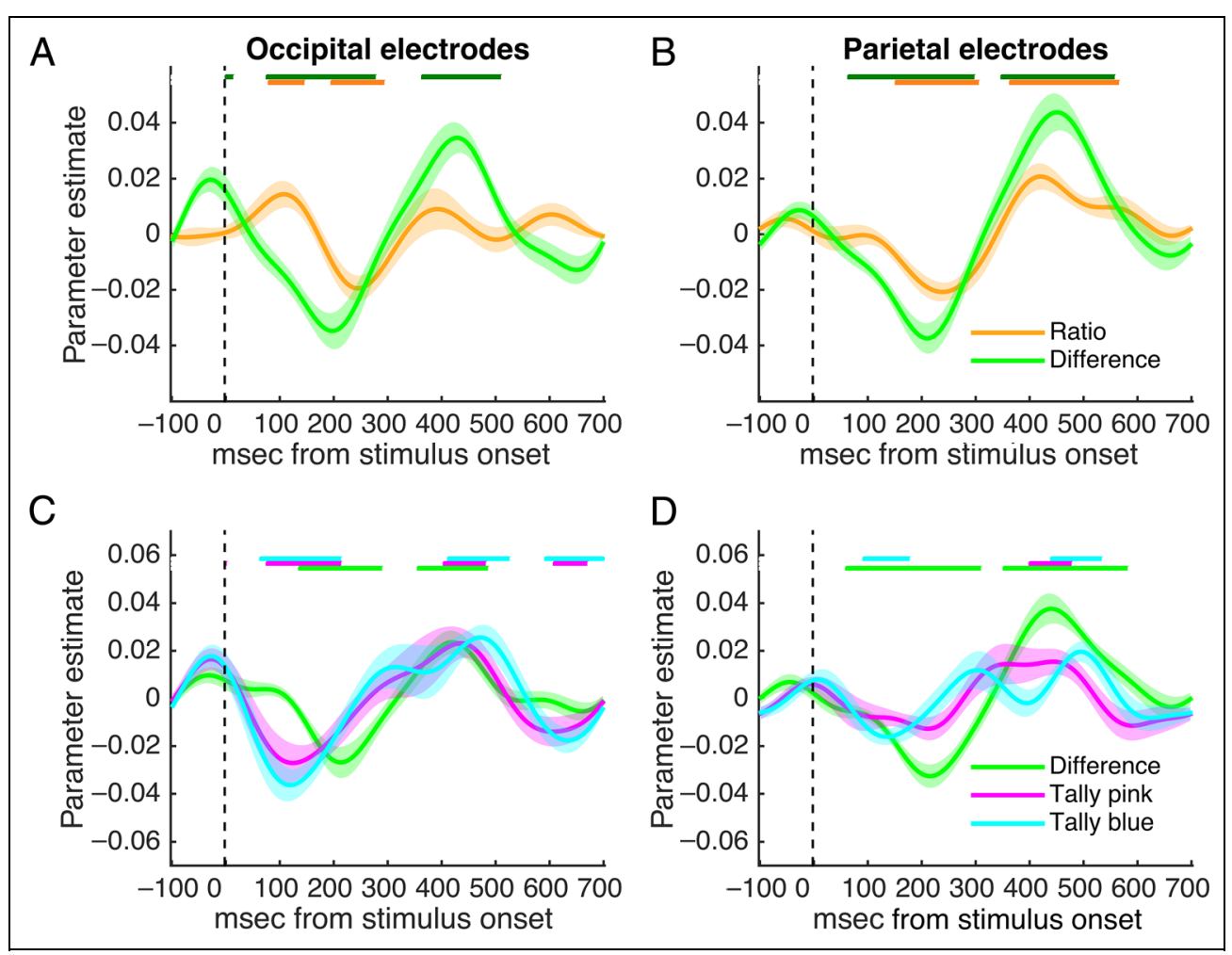


Figure 4. Scalp topographies. Scalp-wide significant correlations between the EEG signal predicted by the tally model (top row) and difference model (bottom row). Note that the tally model predicts that two quantities are encoded (number of pink balls and number of blue balls); these two quantities have been combined in this figure for ease of viewing, but plotting them separately yields a qualitatively similar pattern. The plots show the $t$ values corresponding to times and regions at which (on average in that time bin) the correlation for each model deviated significantly from zero.

To support this notion further, decoding analyses-which are able to link the psychological variable (human choice) with the physiological variable (neural encoding of DU in the run up to choice) - were used to assess the relationship between the residual variance not accounted for by the encoding regressions and human choice (see Methods). This technique allows the link between the neural transformation of DU and choice behavior ("choice probabilities") to be made more explicit. Decoding analysis asks how residual variance in the encoding (described above) of model-predicted quantities impacts choices, over and above the influence of stimulus choices. Although the decoding analysis for the tally model showed no significant effect (Figure 5A), the time courses of the decoding regressions for $\mathrm{DU}_{\text {difference }}$ were in accordance with those seen in encoding in parietal electrodes (Figure 5B). This analysis therefore provides support for the difference model over the tally model and suggests that variation in encoding of the difference signals is predictive of participants' responses. We note that this finding also rules out spurious explanations for the observed decision encoding curves, such as the view that the apparent relationship between decision information and brain activity is somehow secondary to differences in attention or arousal.

\section{EEG: Lateralized Beta-Band Activity}

Previous studies have observed that oscillatory activity in the beta-band range accompanies the buildup of information to a decision threshold (O'Connell, Dockree, \& Kelly, 2012). Next, thus, we measured how lateralized beta-band activity over the motor cortex varied with the cumulative decision evidence in favor of either choice. To this end, we wavelet-transformed EEG data into its spectral components in 10 logarithmically spaced frequency bands between 9 and $43 \mathrm{~Hz}$ (i.e., encompassing the approximate beta-band range extending into lower gamma). First, we confirmed that lateralized beta-band activity was present in the preparation of the motor response (made from 3500 msec after the onset of the first stimulus in each trial) by computing the interhemispheric difference in EEG activity in the 9- to 43- $\mathrm{Hz}$ range between the lateral central electrodes (see Methods). The results confirmed that the difference in power in preparation for responding "pink" (the choice made with the right index finger) minus responding "blue" (made with the left index finger) was positive in the contralateral hemisphere and negative in the ipsilateral hemisphere (Figure 6A), with a focus between $\sim 20$ and $\sim 30 \mathrm{~Hz}$.
Figure 5. Neural decoding curves. The unexplained variance (i.e., the residual error) in the EEG signal from the encoding regressions in parietal regions for the tally model (purple curve) and the difference model (green) were used as predictors of choice in the decoding regressions, allowing us to link neural activity (in parietal electrodes) with behavior choice. See Methods for more detail on this procedure. Shaded areas in the curves denote SEM. The dark green bar shows periods in which the correlation significantly deviated from zero in the parietal signal; note that, for the tally model, there was no such period of significance.

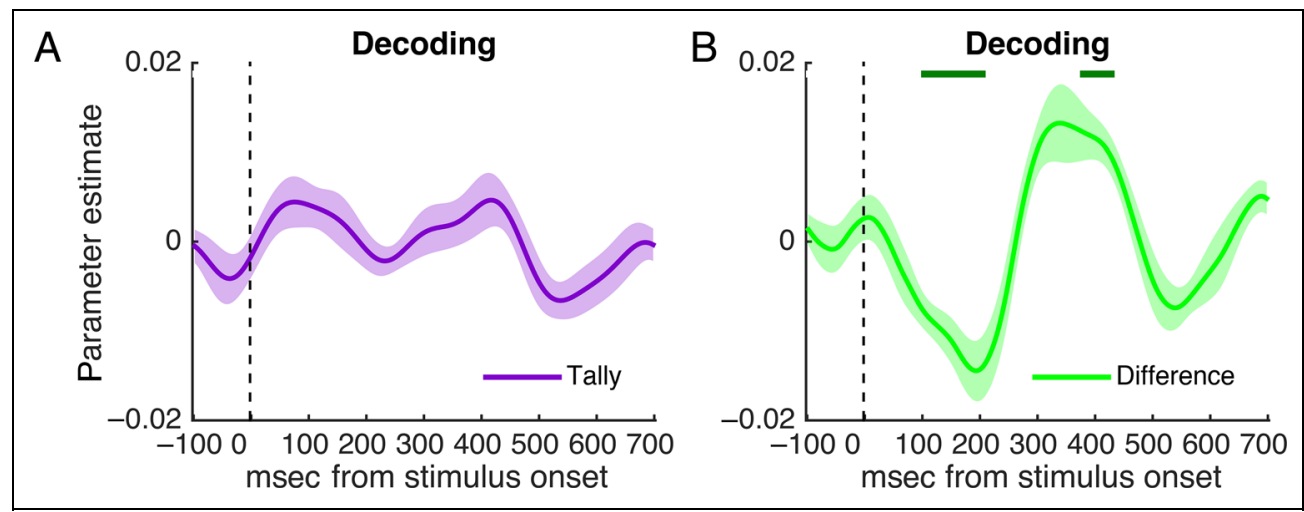


Figure 6. Time-frequency analyses. Interhemispheric difference in log spectral power at 9-43 Hz (i.e., mainly overlapping the beta band frequency, approx. 15-30 Hz) between "blue" choices, which were always made with the right index finger, and "pink" choices, made with the left index finger. The resulting quantity was positive in the contralateral hemisphere to the hand with which the choice was made and negative in the ipsilateral hemisphere. The period displayed is $2.6-3.2 \mathrm{sec}$ after first stimulus onset, that is, close to execution of motor response. Tally and difference model DVs were generated by computing the cumulative sum of the model-derived sampleby-sample quantities $\mathrm{DU}_{\text {tally }}$ and $\mathrm{DU}_{\text {difference, }}$ respectively, across the trial. Note that the tally model predicts that two quantities are encoded (number of pink balls and number of blue balls); these two quantities have been combined in this figure for ease of viewing. The resulting predictors were then regressed against the log spectral power difference between centroparietal electrodes C3 + CP3 and C4 + $\mathrm{CP} 4$, that is, a motor region that should correspond with motor preparatory activity. B shows the correlation between the predictions of the cumulative tally DV and the difference in power between the two hemispheres, and $\mathrm{C}$ depicts the same but in relation to the difference model DV. D and E show times/frequencies at which the respective quantities plotted in B and C deviate significantly from zero.

Finally, we tested whether beta-band activity correlated with the accumulated decision-relevant evidence predicted by each of the models. To this end, we conducted further regression analyses in which the momentary ( $\left.\mathrm{DU}_{\text {difference }}\right)$ and cumulative $\left(\mathrm{DV}_{\text {difference }}\right)$ predictions, as well as the momentary $\left(\mathrm{DU}_{\text {tally }}\right)$ and cumulative $\left(\mathrm{DV}_{\text {tally }}\right)$ predictions, were both entered as predictors of the interhemispheric difference in lateralized log power in the 10 selected frequency bands (see Methods). Note that, for these analyses, we used the signed decision quantities (not rectified) to predict the leftwards versus rightward response. Consistent with the view that participants solve the task by integrating a difference signal, the cumulative difference signal $\mathrm{DV}_{\text {difference, }}$, but not the cumulative tally signal $\mathrm{DV}_{\text {tally }}$, was a reliable predictor of beta-band lateralization over the motor cortex: Figure $6 \mathrm{~B}$ and $\mathrm{C}$ depict how lateralized beta-band power depends on the cumulative $\mathrm{DV}_{\text {tally }}$ (Figure 6B) and $\mathrm{DV}_{\text {difference }}$ (Figure 6C). The relationship with $\mathrm{DV}_{\text {difference }}$ (Figure $6 \mathrm{E}$ ), unlike that with $\mathrm{DV}_{\text {tally }}$ (Figure $6 \mathrm{D}$ ), showed a period of significance from 100 msec onward.

\section{Control Analyses}

Next, to ensure further that the neural responses were driven by decision-relevant information rather than other low-level factors, we measured how both behavior and brain activity correlated with the number of white distractor dots in comparison with pink and blue dots. These analyses allowed us to distinguish the behavioral and neural response to decision information (blue and pink dots) from that elicited by task-irrelevant sensory input 
(distractor white dots). Entering these quantities (white, blue, and pink dots) together into the regression ensured that they competed for unshared variance. In the behavior data, the response to white dots was minimal in comparison with that evoked by reliability-weighted colored dots (Figure 7A). In the neural data, the time course of the resulting regression coefficients showed that the number of white (distractor) dots drove a visual response (Figure 7C and D, gray curve) peaking around $250 \mathrm{msec}$ post-stimulus. Although the neural encoding of the white dots was significant, it followed a qualitatively different pattern from the encoding responses to the decision-relevant quantities (pink and blue dots; indicated in Figure $7 \mathrm{C}$ and $\mathrm{D}$ by pink and blue curves, respectively) in both parietal and occipital electrodes. Combined with the behavior analysis showing limited effect of white dots on choice, the qualitatively different neural encoding pattern suggests that the white dots were being processed via a different, decision-irrelevant mechanism. The responses to the colored dots were characterized by negative and positive deflections peaking at $\sim 100$ and $\sim 400 \mathrm{msec}$ post-stimulus, respectively.

We also examined the relationship between brain activity and the difference in magnitude (i.e., the total number of dots on each sample, both decision relevant [blue and pink] and decision irrelevant [white]) from one sample to the next, regressing the absolute difference in magnitude from sample to sample against the EEG signal over parietal electrodes (see Methods). Perhaps surprisingly, we found no correlation between the change in magnitude and parietal EEG signals, with no time window reaching statistical significance (Figure $7 \mathrm{E}$ ). This suggests that our neural data are dominated by decision-related effects rather than the influence of passive adaptation to number.

\section{Control Experiment}

Finally, to determine whether the observed effects of sample size were driven by the number of dots, rather than low-level visual signals such as the total pixel area taken up by the colored dots, we conducted a further control experiment. In this experiment, a new cohort of participants ( $n=19$ ) performed the same urn choice task, with one difference: The pink and blue dots were different sizes, ensuring that the pixel area taken up by the dots and the number of dots were dissociable on each sample, for each color individually (correlation between number of pink dots and pink pixel area: $r=.65$, correlation between number of blue dots and blue pixel area: $r=.66)$ and overall without white dots $(r=.68)$ and with white dots $(r=.65)$. This enabled us to investigate sample size effects using the same regression approach, in which we predicted choices as a function of the ratio of dots, sorting samples into predictors by their sample size (see above) but now partialing out that portion of the variance that could be ascribed to pixel area.
To achieve this, we entered the sample-wise pixel area for blue and pink categories as additional regressors into the design matrix. The resulting coefficients could be interpreted in the same fashion as in Figure $2 \mathrm{C}$. The results show that, although decisions are partly influenced by number of pixels, the sample size effect on dots remains robust to the inclusion of these additional regressors (Figure 7B), following the same pattern of increasing weight on choice with increasing sample size as seen in the previous analyses (cf. Figure 2C). This was confirmed by an ANOVA on the weights over ranks: for the weights corresponding to sample size, $F(7,144)=32.6, p<.001$. As before, the beta weight in the human data for the largest samples was significantly higher than that of the smallest samples: $t(18)=7.8, p<.001$. However, the same was not the case for the beta weights corresponding to pixel area: Although the weights deviated significantly from zero, the weights did not deviate significantly from each other, unlike the weights corresponding to sample size $(F=0.71, p=.66$ for blue pixel area; $F=0.67, p=.7$ for pink pixel area).

\section{DISCUSSION}

Humans making decisions are often faced with information that is indistinct, weak, or ambiguous. During sensorimotor choices, humans rely more heavily on inputs coming from sources that are clearly discernable, for example, by weighting haptic information over visual information when the latter has been corrupted by noise (Ernst \& Banks, 2002). In one sense, it is remarkable that human behavior has been optimized over evolutionary history to weight information by its reliability, as a statistically ideal observer should. On the other hand, it is perhaps natural that human decisions rely more on information that can be easily detected than that which cannot. Indeed, uncertainty can arise at multiple stages during information processing, and so decision-relevant evidence can be rendered unreliable by other means than corruption from lowlevel input noise. Consider, for example, a juror evaluating evidence in a court of law. On the one hand, a witness whose testimony is rendered incomprehensible will be unlikely to hold much sway over the jury, compared with one that can be clearly understood (uncertainty because of lowlevel sensory noise). However, even where testimony is clearly perceptible, a juror might question whether a lone witness (rather than, say, 10 consistent testimonies) is sufficient to condemn the accused (uncertainty because of high-level knowledge of sample size). Here, we tested whether humans integrate information optimally when uncertainty arises at a higher, rather than lower, level. Unlike previous studies using description-based scenarios, we found that participants automatically weighed the information by sample size, behaving in a fashion that resembled an ideal observer.

Previous research has revealed that humans often disregard the quantity or quality of information when 


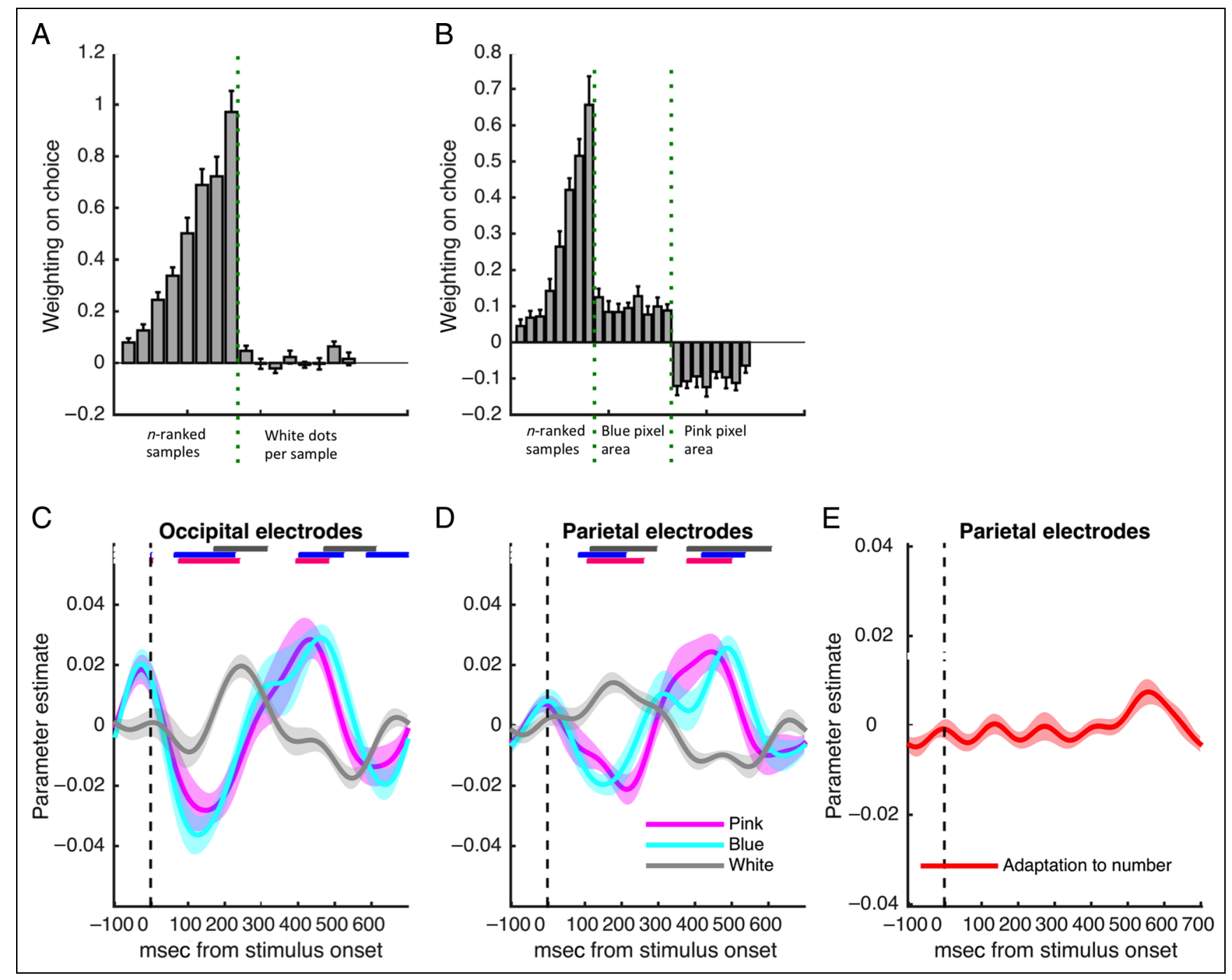

Figure 7. Control analyses. The first eight gray bars show weights (beta coefficients) given to the colored (decision-relevant) dots in each sample, ranked by sample size (smallest to largest). The second set of eight gray bars show the weight given to white (decision-irrelevant) dots in each sample. The response to the colored dots clearly outweighs the response to the white dots. (B) Gray bars depict the weight (beta coefficients) on choosing "blue" of various aspects of the decision array. The first set of eight bars show weights given to each sample as ranked by sample size (smallest to largest), the second set of eight bars show the weight of total pixel area of blue dots per sample, and the final set of eight bars show the weight of the total pixel area of pink dots per sample, on choosing "blue." All quantities were entered into the same (probit) regression to ensure they competed for unshared variance. In all, black bars denote SEM. (C and D) Correlation between decision-relevant (blue and pink dots) and decision-irrelevant (white dots) information and the EEG signal in occipital electrodes (C) and parietal electrodes (D). Shaded areas in the curves denote SEM. The bars at the top show periods in which the curves consistently deviate significantly from zero across participants and trials; the colors of these bars correspond to the colors of the curves they reflect. (E) We regressed a quantity corresponding to the difference in number of dots (blue + pink + white) between one sample and the next against the EEG signal over parietal electrodes. The curve shows that the correlation between this quantity and the neural activity was small with no time points reaching significance. Shaded areas in the curve denote $S E M$. This suggests that the parietal encoding signal (see Figure 3) is indeed likely to reflect decision-related effects, rather than the influence of passive adaptation to number.

making judgments (Griffin \& Tversky, 1992). This "sample size neglect" can be considered as a special case of a more general bias by which humans neglect the duration or extent over which information is available (Kahneman et al., 1982). However, this classic research presented decision problems in the form of descriptive scenarios, making it unclear whether optimality was limited by the format of decision information (description vs. experience based) or by the locus of uncertainty (low-level vs. high-level uncertainty). Here, we assessed human decisions using an expanded judgment task in which discrete samples of information arrived in sequence, after which participants made a categorical judgment about their provenance. We found that participants paid more heed to samples that offered better quality information: For example, decisions were more influenced by samples on which there were 12 balls, rather than three balls, even if the ratio of blue to pink balls was 2:1 in both cases. In other words, this classic demonstration of suboptimal behavior from the decisionmaking literature may owe more to the format in which information was presented, rather than the locus of uncertainty in information processing. This has important 
implications for a range of real-word situations, including medicine, economics, and public policy, where sample size neglect might lead to poor or biased decisions.

The optimal solution to the urn-and-balls problem described here is to estimate the binomial odds ratio that the balls were drawn from either of the two urns and decide according to the sign of its logarithm. Note that samples were presented at $3 \mathrm{~Hz}$, too fast to rely on explicit mathematical calculation; any integration must be of approximate number or magnitude (Piazza \& Izard, 2009). Thus, although humans behaved near-optimally, it is implausible that they were explicitly computing binomial probabilities to solve the task. Rather, there are two mechanisms that arrive at the binomial solution that participants could have used. The first is simply to tally up the approximate number of pink and blue dots independently and respond whichever is greater (tally model). The second is to estimate the approximate difference between pink and blue dots on each sample and respond according to whether it is greater or less than zero (difference model). Where the experimenter, rather than the observer, determines the viewing time that precedes choices (as in this experiment), these models make very similar predictions (identical, except for added stochasticity) regarding behavioral data but differ in their neural predictions. The computational approaches described by the tally and difference models are related respectively to the "race" and "diffusion" accounts of the integration process that is a prelude to human categorical choices (Ratcliff \& McKoon, 2008; Vickers, 1979).

The tally model requires that independent totals of blue and pink dots are registered, but not their relative difference. Nevertheless, we witnessed neural signals over the parietal cortex that scaled with the difference in the total number of blue and pink dots, even when other confounding factors had been taken into account. This supports the difference model but not the tally model. Indeed, a closer look at the encoding of information over occipital electrodes suggested that the number of blue and pink dots was encoded early (100 msec), followed by the difference signal, exactly as if the brain first estimated independent totals for each sample and then compared them. This is reminiscent of the successive encoding of absolute and relative economic value in magnetoencephalographic activity observed during a gambling task (Hunt et al., 2012). In the parietal cortex, the decision information predicted by the difference model exhibited a temporal profile characterized by an early dip (at $\sim 220$ msec post-stimulus) and a later peak (at $\sim 450 \mathrm{msec}$ ). The same pattern of EEG activity has previously been shown to scale with decision information in a task involving discrimination of the mean orientation of a stream of tilted gratings, with a negative deflection at $\sim 250 \mathrm{msec}$ and a positive peak at $\sim 500 \mathrm{msec}$ after each sample (Wyart, Nobre, et al., 2012).

A wealth of research in humans and nonhuman primates has implicated the parietal cortex in the representation of approximate number (Piazza \& Izard, 2009). At the single- cell level, individual neurons show bell-shaped tuning curves over the number line (Nieder \& Miller, 2003). In human imaging studies, neural signals localized to the parietal cortex scale with the numerical disparity between two successively or simultaneously occurring stimuli (i.e., sets of dots), even in the absence of an overt estimation task (Piazza et al., 2004). The focus of our experiment was not to distinguish accounts based on number from those based on magnitude per se. Nevertheless, it is unlikely that our neural findings simply reflect low-level adaptation to number or magnitude, for four reasons. First, a control experiment in which the pixel area taken up by the colored dots and the number of colored dots were decorrelated still showed a strong sample size effect, even when the lower level factor (pixel area) was included as a nuisance covariate in the regression. Second, the gain of encoding of number was qualitatively different for blue and pink stimuli, which were decision relevant, than for white stimuli, which were not. Third, our decoding analysis suggested that the strength with which neural signals encoded the difference in number of pink and blue dots was predictive of later choices, as would be expected of a decision signal. Fourth, we observed a distinct neural signal over motor regions that reflected the buildup in magnitude differences over time. These findings suggest that the parietal signal observed here instead reflects the relative difference between decision information in favor of either category. One way of linking our findings with this literature is to assume that, in the absence of an overt task, participants implicitly compare information arriving in sequence, calculating their difference as an implicit decision signal. Indeed, a similar pattern of adaptation to numerosity is obtained when participants make same-different judgments on successive stimuli. In other words, the representation of numerosity in the parietal cortex might reflect a more general representation of the magnitude of the information relevant for a decision (Wyart, Nobre, et al., 2012).

In summary, we show that humans integrate approximate number in a near-optimal (reliability-weighted) fashion. Our study has implications for research in a number of domains, including economics and medical diagnosis, in which it is widely assumed that humans estimate numerical quantities in a biased and suboptimal fashion. Humans can make near-optimal judgments about values or other numerical estimates if the information is presented in an appropriate format.

\section{Acknowledgments}

We thank Nick Yeung for providing access to EEG equipment. This work was funded by an ERC Starter grant to C. S.

Reprint requests should be sent to Christopher Summerfield, Department of Experimental Psychology, University of Oxford, South Parks Road, Oxford, United Kingdom of Great Britain and Northern Ireland, OX1 3UD, or via e-mail: christopher. summerfield@psy.ox.ac.uk. 


\section{REFERENCES}

Brainard, D. H. (1997). The psychophysics toolbox. Spatial Vision, 10, 433-436.

Delorme, A., \& Makeig, S. (2004). EEGLAB: An open source toolbox for analysis of single-trial EEG dynamics including independent component analysis. Journal of Neuroscience Methods, 134, 9-21.

Ernst, M. O., \& Banks, M. S. (2002). Humans integrate visual and haptic information in a statistically optimal fashion. Nature, 415, 429-433.

Gitelman, D. R., Penny, W. D., Ashburner, J., \& Friston, K. J. (2003). Modeling regional and psychophysiologic interactions in fMRI: The importance of hemodynamic deconvolution. Neuroimage, 19, 200-207.

Gold, J. I., \& Shadlen, M. N. (2007). The neural basis of decision making. Annual Review of Neuroscience, 30, 535-574.

Gould, I. C., Nobre, A. C., Wyart, V., \& Rushworth, M. F. (2012). Effects of decision variables and intraparietal stimulation on sensorimotor oscillatory activity in the human brain. Journal of Neuroscience, 32, 13805-13818.

Griffin, D., \& Tversky, A. (1992). The weighting of evidence and the determinants of confidence. Cognitive Psychology, 24, 411-435.

Hertwig, R., \& Erev, I. (2009). The description-experience gap in risky choice. Trends in Cognitive Sciences, $13,517-523$.

Hunt, L. T., Kolling, N., Soltani, A., Woolrich, M. W., Rushworth, M. F., \& Behrens, T. E. (2012). Mechanisms underlying cortical activity during value-guided choice. Nature Neuroscience, 15, 470-476.

Josephs, O., Turner, R., \& Friston, K. (1997). Event-related fMRI Human Brain Mapping, 5, 243-248.

Kahneman, D., Slovic, P., \& Tversky, A. (1982). Judgment under uncertainty: Heuristics and biases. New York: Cambridge University Press.

Kording, K. P. (2007). Decision theory: What "should" the nervous system do? Science, 318, 606-610.

Kording, K. P., \& Wolpert, D. M. (2004). Bayesian integration in sensorimotor learning. Nature, 427, 244-247.

Maris, E., \& Oostenveld, R. (2007). Nonparametric statistical testing of EEG- and MEG-data. Journal of Neuroscience Methods, 164, 177-190.
Nieder, A., \& Miller, E. K. (2003). Coding of cognitive magnitude: Compressed scaling of numerical information in the primate prefrontal cortex. Neuron, 37, 149-157.

Nienborg, H., \& Cumming, B. (2010). Correlations between the activity of sensory neurons and behavior: How much do they tell us about a neuron's causality? Current Opinion in Neurobiology, 20, 376-381.

O'Connell, R. G., Dockree, P. M., \& Kelly, S. P. (2012). A supramodal accumulation-to-bound signal that determines perceptual decisions in humans. Nature Neuroscience, 15, 1729-1735.

Piazza, M., \& Izard, V. (2009). How humans count: Numerosity and the parietal cortex. The Neuroscientist, 15, 261-273.

Piazza, M., Izard, V., Pinel, P., Le Bihan, D., \& Dehaene, S. (2004). Tuning curves for approximate numerosity in the human intraparietal sulcus. Neuron, 44, 547-555.

Pouget, A., Beck, J. M., Ma, W. J., \& Latham, P. E. (2013). Probabilistic brains: Knowns and unknowns. Nature Neuroscience, 16, 1170-1178.

Ratcliff, R., \& McKoon, G. (2008). The diffusion decision model: Theory and data for two-choice decision tasks. Neural Computation, 20, 873-922.

Smith, P. L., \& Vickers, D. (1988). The accumulator model of two-choice discrimination. Journal of Mathematical Psychology, 32, 135-168.

Smith, P. L., \& Vickers, D. (1989). Modeling evidence accumulation with partial loss in expanded judgment. Journal of Experimental Psychology: Human Perception and Performance, 15, 797-815.

Tversky, A., \& Kahneman, D. (1971). Belief in the law of small numbers. Psychological Bulletin, 76, 105-110.

Vickers, D. (1979). Decision processes in visual perception. London: Academic Press.

Wyart, V., de Gardelle, V., Scholl, J., \& Summerfield, C. (2012). Rhythmic fluctuations in evidence accumulation during decision making in the human brain. Neuron, 76, 847-858.

Wyart, V., Myers, N. E., \& Summerfield, C. (2015). Neural mechanisms of human perceptual choice under focused and divided attention. Journal of Neuroscience, 35, 3485-3498.

Wyart, V., Nobre, A. C., \& Summerfield, C. (2012). Dissociable prior influences of signal probability and relevance on visual contrast sensitivity. Proceedings of the National Academy of Sciences, U.S.A., 109, 3593-3598.

Yang, T., \& Shadlen, M. N. (2007). Probabilistic reasoning by neurons. Nature, 447, 1075-1080. 